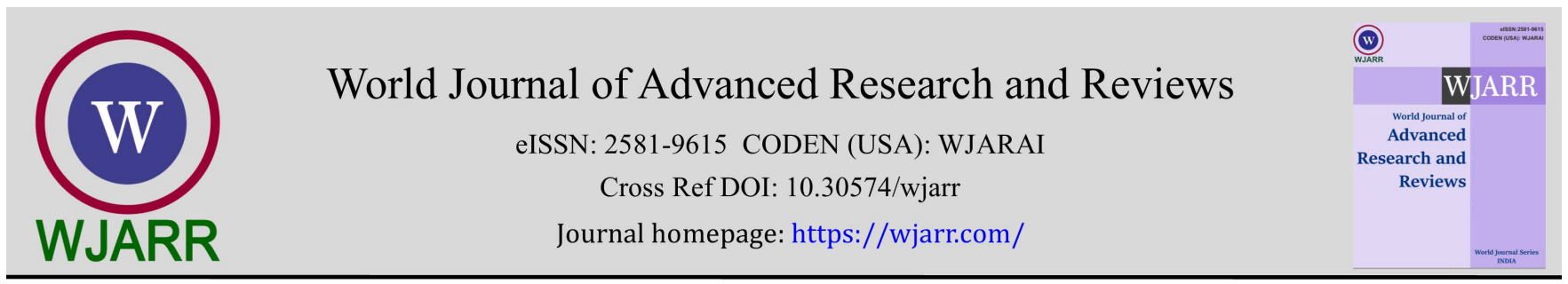

(REVIEW ARTICLE)

\title{
Changes in profibrotic activity in cardiovascular diseases
}

\author{
Ivaneta D Yoncheva ${ }^{1,}{ }^{*}$, Denislav E Biserov ${ }^{1}$ and Maria N Negreva MD ${ }^{2}$ \\ 1 University Hospital Virgin Mary, Burgas, Bulgaria. \\ 2 University Hospital St. Marina, Varna, Bulgaria.
}

World Journal of Advanced Research and Reviews, 2021, 11(02), 093-099

Publication history: Received on 02 July 2021; revised on 04 August 2021; accepted on 06 August 2021

Article DOI: https://doi.org/10.30574/wjarr.2021.11.2.0368

\begin{abstract}
The myocardium consists of several types of cells: cardiomyocytes, cardiac fibroblasts, endothelial cells and smooth muscle cells. Fibroblasts are cells of mesenchymal origin and are present in all tissues in the body. Cardiac damage can activate available CFBs, provoke transformation of endothelial or epithelial cells into fibroblasts, or induce the production of CFBs from hematopoietic cells and bone marrow. The change in ECM is a key point in the remodeling of the heart in response to the disease process. Disruption of the reticular structure of the ECM alters the connection between myocardial cells and blood vessels, thereby disrupting the structure and function of the heart muscle. Type I and III collagen fibrils are the predominant part of the ECM of the heart. They are synthesized as procollagen, which is converted to a mature collagen molecule. Procollagen type I propeptide (PICP), amino-terminal propeptide type I procollagen (PINP) and N terminal type III collagen peptide (PIIINP) are released in proportional amounts in the synthesis of collagen types I and III and can be used as serum markers for these processes. On the other hand the differentiation from CFBs to myoFB is supported by the transforming growth factor beta (TGF- $\beta$ ), connective tissue growth factor (CTGF), a number of cytokines in the ECM and others. The scientific community is faced with the question of which biomarkers to use to identify the early stages of development of cardiac fibrosis, as well as how to assess the degree of progression of this pathological process.
\end{abstract}

Keywords: Cardiac fibroblasts; Procollagen; Cytokines; Cardiac damage

\section{Introduction}

The myocardium consists of several types of cells: cardiomyocytes, cardiac fibroblasts, endothelial cells and smooth muscle cells. A study by Nag, as early as 1980, quantified the cellular content of heart tissue from rats using an electron microscope [1]. He found that the heart muscle consists of 70\% non-myocyte cells and 30\% cardiomyocytes.

Fibroblasts are cells of mesenchymal origin and are present in all tissues in the body. In the myocardium, they are called cardiac fibroblasts (CFBs) and are unique, in comparison to other cell types, because they do not have a basement membrane.

With appropriate stimulation, CFBs can be transformed into myofibroblasts (myoFBs), which are more mobile and contractile, and also have increased secretory activity in order to produce extracellular matrix (ECM) proteins [2]. MyoFBs, discovered and named by Gabbiani in 1971, are not found in a healthy myocardium, they appear in the presence of myocardial damage.

MyoFBs play a key role in the development of fibrous tissue in the area of myocardial infarction, as well as in a number of other pathological processes leading to tissue damage [3]. The differentiation from CFBs to myoFB is supported by

\footnotetext{
* Corresponding author: Ivaneta D Yoncheva

University Hospital Virgin Mary , Burgas , Bulgaria.

Copyright (C) 2021 Author(s) retain the copyright of this article. This article is published under the terms of the Creative Commons Attribution Liscense 4.0.
} 
the transforming growth factor beta (TGF- $\beta$ ), connective tissue growth factor (CTGF), a number of cytokines in the ECM and others.

Cardiac damage can activate available CFBs, provoke transformation of endothelial or epithelial cells into fibroblasts, or induce the production of CFBs from hematopoietic cells and bone marrow [4]. Development of myocardial fibrosis is a result of a pathological response to the pressure-loaded heart muscle and occurs with proliferation of available CFBs, as well as transformation of endothelial cells into mesenchymal and production of CFBs and myoFBs.

There are two types of myocardial fibrosis: replacement and interstitial [5]. Replacement fibrosis develops in the area of the scar after myocardial infarction. It can also occur in hypertrophic cardiomyopathy, sarcoidosis, myocarditis, chronic renal failure and toxic cardiomyopathy. Interstitial fibrosis is a diffuse process, which affects the myocardium and can be reactive and infiltrative. Reactive fibrosis manifests in a number of diseases, including hypertension and agerelated increase in fibrous deposition. Infiltrative fibrosis is relatively rare and is caused by progressive interstitial deposition of insoluble proteins in amyloidosis or glycosphingolipids in Anderson-Fabry disease [6].

Both types of fibrosis, interstitial and infiltrative, can lead to apoptosis of cardiomyocytes and development of connective tissue deposition. Regardless of the type, myocardial fibrosis is a complex process leading to changes in quantity and composition of ECM.

The change in ECM is a key point in the remodeling of the heart in response to the disease process. Disruption of the reticular structure of the ECM alters the connection between myocardial cells and blood vessels, thereby disrupting the structure and function of the heart muscle. On the other hand, increased production and accumulation of structural proteins in the ECM or development of fibrosis leads to increased myocardial stiffness, thus compromising its contraction and relaxation. Collagen overproduction and subsequent fibrosis is a clear cause of increased myocardial stiffness, resulting in systolic and diastolic dysfunction.

\section{Collagen in the ECM}

Type I and III collagen fibrils are the predominant part of the ECM of the heart. They are synthesized as procollagen, which is converted to a mature collagen molecule by cleavage of a propeptide region through procollagen peptidase.

Grouping and twisting of mature collagen molecules gives rise to collagen fibrils and subsequently fibers. During physiological metabolism in the ECM or in pathological remodeling processes, collagen fibers undergo degradation by cleavage of the telopeptide from the amino-terminal or carboxy-terminal end of the collagen molecule. Propeptides from the carboxy-terminal or amino-terminal region of type I collagen (PICP, PINP) and those from type III collagen (PIIICP, PIIINP) are released into the bloodstream in proportional amounts and are biomarkers for collagen synthesis. On the other hand, the carboxy-terminal or amino-terminal telopeptide region of type I collagen (CITP, NITP) and type III (CIIITP, NIIITP) are produced and are biomarkers for its degradation.

The types of collagen that are present in myocardial tissue in greater quantities are type I and III [7]. Type I has low specificity, but represents the majority of cardiac collagen (85\%), providing tensile strength and resistance to tension and deformation. Type III is less represented, but much more specific to the heart and provides elasticity.

Collagen fibrils in the myocardium are a substrate for matrix metalproteinases (MMPs), with MMP-1 having the highest affinity and degrading mainly type I and III collagen. Metalproteinase activity depends on the relative concentration of the active enzyme and presence of a tissue inhibitor of MMPs called TIMPs. These two enzymes are simultaneously present in cardiac fibroblasts, and their fine regulation is the basis for maintaining the architecture of ECM [8]. Changes in ECM balance can lead to cardiac fibrosis. Fibrosis, in its turn, is crucial for the development of diastolic dysfunction, impaired pumping capacity and may be a structural substrate for arrhythmogenicity, development and progression of congestive heart failure (HF) and sudden death. Proliferation and phenotypic transformation of fibroblasts is associated with a change in MMPs expression and is a major process in ventricular remodeling in the pathophysiology of hypertension, myocardial infarction and HF.

As already mentioned, procollagen type I propeptide (PICP), amino-terminal propeptide type I procollagen (PINP) and $\mathrm{N}$ terminal type III collagen peptide (PIIINP) are released in proportional amounts in the synthesis of collagen types I and III and can be used as serum markers for these processes.

Studying these biomarkers in patients with heart diseases may elucidate the process of ECM remodeling in various pathological conditions in the myocardium [9]. 


\section{Role of CTGF and TGF- $\beta$ in the manifestation of cardiac fibrosis}

CTGF, also known as CCN2, is one of the best-studied members of the CCN family, which are regulatory proteins of the ECM [10]. The acronym CCN stands for the first three proteins found in this family: Cysteine rich angiogenic inducer 61 (CYR61), CTGF and Nephroblastoma overexpressed protein (NOV).

CTGF is normally expressed in minimal amounts, but its concentration increases dramatically in profibrous conditions [11].

It has been shown in cell culture studies, that CTGF regulates multiple processes leading to fibrogenesis and cell proliferation. This occurs with participation of TGF- $\beta$ and affects various cells involved in fibrogenesis, such as mesenchymal stem cells, glomerular podocytes, mesangial cells, lung type II alveolar cells, vascular smooth muscle cells, endothelial cells, cardiomyocytes, fibroblasts and others. During embryogenesis, CTGF is expressed in larger amounts, participating in the development of the kidneys, cardiovascular system and skeleton. In adults, its expression is minimal, and at transcriptional level is induced by stimuli such as growth factors, cytokines, hormones, mitogens, ultraviolet light, hypoxia and mechanical stress [12].

Correlation studies with pathologically altered human cells also show a strong connection between CTGF and TGF- $\beta$ and a number of diseases including diabetic nephropathy, pulmonary fibrosis, liver fibrosis, atherosclerosis, congestive heart failure, pancreatitis and some forms of malignancies.

TGF- $\beta$ is a major regulator of tissue growth, regeneration, remodeling and fibrosis [13]. This is associated with close interaction with CTGF, as well as stimulation of processes in the ECM such as proliferation of fibroblasts, osteoblasts and astrocytes and fibrosis in healing processes.

Cardiac fibrosis is most common in the presence of hypertensive heart disease and diabetes mellitus. It is a process that affects a large percentage of cardiovascular patients, and at the same time, there are no major therapeutic options at the moment. CTGF is expressed in cardiac fibroblasts and in cardiomyocytes, and this process is enhanced by TGF- $\beta$. This leads to increased synthesis of ECM components such as fibronectin, collagen type I and III. These changes have been established in animal models with a history of myocardial infarction, and these growth factors are found in high quantities in the connective tissue replacing the necrotic area [14,15].

\section{High cardiovascular risk profile and cardiac fibrosis}

Over weight is associated with major cardiovascular risk factors such as diabetes mellitus, hypertension and dyslipidemia. A number of studies have found a connection between obesity and increased cardiac fibrosis. Overweight is associated not only with development of metabolic risk factors for atherosclerosis, but is also strongly associated with left ventricular hypertrophy, HF, atrial fibrillation (AF) and stroke. Changes in the levels of procollagen type III, metalproteinases and their tissue inhibitors have been established, and these changes correlate with manifestation of diastolic dysfunction [16]. Increased interstitial fibrosis in the atrial myocardium has also been found in experimental animal models with obesity. These structural changes are associated with changes in the conductive properties of the LA myocardium, which increases the risk of developing arrhythmias. This coincides with data from epidemiological studies, where obesity is an established risk factor for manifestation of AF [17].

Increased body weight is associated with increased myocardial oxygen consumption, increased myocardial fatty acid metabolism, and decreased efficiency of myocardial contraction $[18,19]$. There is evidence to suggest a strong link between obesity, development of diastolic dysfunction, left ventricular hypertrophy and left atrial strain. On the one hand, this is due to increased minute volume, increased peripheral vascular resistance and development of left ventricular hypertrophy. Elevated concentrations of free fatty acids in obese patients have a cardiotoxic effect, which is also related to the development of LV hypertrophy.

Obesity has been shown to increase TGF- $\beta$ activity [20]. It has three isoforms, the most important being type 1, which is known to be expressed in human adipose tissue and its levels correlate with BMI.

TGF- $\beta$ overexpression is associated with isolated atrial fibrosis, myocardial heterogeneity, and increased AF manifestation in transgenic mice [21]. TGF- $\beta$ and CTGF induce the transformation of fibroblasts into myofibroblasts. This induces extracellular gene expression for increased collagen synthesis and at the same time inhibits gene expression for MMPs. 
Changes in cardiac tissue composition have been observed in patients with left ventricular hypertrophy in hypertension and heart failure and lead to structural remodeling of the myocardium [22]. One of these changes is imbalance between the synthesis and degradation of type I and III collagen, leading to increased myocardial collagen content. Myocardial fibrosis is a consequence of a number of pathological processes mediated by mechanical, neurohormonal and cytokine pathophysiological processes. The clinical significance of fibrosis is that it can lead to HF and other complications in patients with hypertensive heart disease.

Nakahara et al. found that serum PIIINP concentrations significantly correlated with relative wall thickness (RWT) in hypertensive patients with overt left ventricular hypertrophy [23]. Interestingly, although the subgroups with eccentric and concentric hypertrophy did not show differences in LVMI and BNP, the serum concentration of PIIINP was significantly higher in those with concentric hypertrophy. The authors then suggested that aldosterone, whose concentration in blood is associated with that of PIIINP, could lead to the development of eccentric hypertrophy by increasing cardiac fibrosis.

Presence of HF in hypertensive patients has been associated with manifestation of diastolic LV dysfunction, caused by changes in myocardial relaxation and stiffness as a result of collagen accumulation [24]. Also, deposition of collagen fibrils in the perivascular areas can lead to reduced vasodilatory capacity of intramyocardial vessels, and hence to reduction of the coronary reserve in the hypertensive heart. Determination of serum levels of markers for the metabolism of type I and III collagen provides indirect information on the extent and ability of antihypertensive treatment to affect myocardial fibrosis. Echocardiography showed a significant association between PIIINP serum levels and decreased longitudinal strain.

As early as 2005, a team of researchers conducted a study in 239 patients with chronic HF, 64 patients with hypertension and diabetes mellitus and compared them with 92 healthy volunteers [25]. Results showed that the markers for collagen synthesis were increased in chronic HF patients, while those for its degradation were reduced. This resulted in subsequent cardiac fibrosis and unfavorable outcome. They also found that changes in collagen metabolism may occur early in high-risk patients, before the clinical manifestation of HF symptoms.

Lofsjogard et al examined the relationship between metabolism biomarkers of type I collagen and clinical symptoms, LV size, systolic and diastolic function, BNP and the manifestation of asynchrony in patients with congestive HF [26]. They found that higher PICP levels were associated with higher BNP levels, greater LV end-diastolic diameter (LVEDd), shorter isovolumetric relaxation time (IVRT), and also a tendency toward more pronounced ventricular asynchrony.

They also found that in higher CITP levels, there were elevated BNP levels, increase in the septal E/e's ratio, and a wider QRS complex.

This proves once again that PICP and CITP levels reflect type I collagen metabolism [27]. The association of these biomarkers with BNP, diastolic function, and LV sizes demonstrates the role of collagen metabolism in the pathophysiology of $\mathrm{HF}$.

Myocardial fibrosis accompanies chronic HF, but its manifestation depends on the etiology of the cardiac dysfunction. It can manifest by prolongation of the QRS complex, frequent ventricular ectopia and ventricular tachyarrhythmias [28].

When fibrosis diffusely affects the heart muscle, it leads to impaired contractility. The degree of myocardial involvement can be assessed by MRI, endomyocardial biopsy, and study of collagen synthesis and degradation serum markers. According to a study conducted by Lopez et al, serum PICP levels correlated with total collagen volume in HF patients in hypertensive heart disease, but not in ischemic disease and idiopathic cardiomyopathy (ICM) [29]. However, PIIINP levels correlated with type III collagen fraction in patients with overt CH in ICD and ICM. Significant disadvantage of both biomarkers is their low specificity. Liver and lung fibrosis, as pathological manifestations of various diseases, can lead to similar increase in these markers.

It is an indisputable fact that fibrous content in tissues and ECM in particular increases with age.

Currently available data indicate that there is an increased expression of CTGF with age, which may be associated with ECM remodeling with aging [30]. This in turn is associated with a number of pathological changes in the blood vessels such as atherosclerosis, aortic dissection, cerebral microhemorrhages and others.

Diabetes mellitus is another disease that is associated with increased cardiovascular risk profile, increased ischemic disease and heart failure manifestation. This leads to reduced survival and impaired quality of life in affected patients. 
Several studies have shown that HF incidence is significantly higher in female patients with diabetes mellitus (DM) [31]. Men with DM are twice as likely to develop HF compared to men without DM, and women with DM have a fivefold increase in that risk. Recently, many studies have focused on the mechanism of development of structural changes in the heart muscle in patients with diabetic cardiomyopathy (DCM) [32].

There is evidence that DCM develops as a result of a number of complex pathophysiological processes, based on chronic hyperglycemia and concomitant hyperlipidemia. This is followed by increased oxidative stress, metabolic disorders, remodeling of the extracellular matrix and cardiac fibrosis. Late manifestations of DCM include changes in cardiac function such as left ventricular hypertrophy, diastolic dysfunction, systolic dysfunction including terminal HF, and death.

This indicates that cardiac fibrosis plays a key role in the pathogenesis of DCM [33]. TGF- $\beta$ has also been found to be one of the mediator molecules involved in the progression of fibrosis by activating intracellular signaling pathways.

Left ventricular hypertrophy is known to be a structural remodeling of the myocardium and is an important marker for cardiovascular disease [34]. An echocardiographic-based study showed that $26 \%$ of normotensive diabetics have elevated left ventricular muscle mass index. This suggests that diabetes provokes left ventricular hypertrophy, regardless of concomitant hypertension and ischemic disease. Diastolic dysfunction is another manifestation of DCM, developing in $40 \%$ of patients [35]. TGF- $\beta$ is one of the most studied mediators. In animal models and in type 1 and 2 diabetes, increased expression of TGF- $\beta$ has been observed, which is associated with cardiac fibrosis [36]. Also, its inhibition can reduce fibrotic changes in the myocardium, which has been proven in animal experiments [37]. This raises the question of new therapeutic strategies to respond to fibrotic changes associated with a number of chronic diseases.

\section{Conclusion}

At first, increased cardiac fibrosis has a protective effect. Persistence of provoking factors, however, leads to negative effects on cardiac function, which over time progresses to manifestation of systolic and diastolic heart failure. Left ventricular hypertrophy also develops as an adaptive mechanism in athletes and during pregnancy. It reverses when the provocative situation subsides and does not progress to heart failure. Persistent left ventricular stress in existing heart disease leads to pathological left ventricular hypertrophy, which is associated with impaired cardiomyocyte function, apoptosis, and cell death.

At present, the gold standard in diagnosis and quantification of interstitial collagen content is endomyocardial biopsy. There are also a number of non-invasive methods for identifying fibrous tissue. MRI with gadolinium-based contrast agents is most commonly used to determine LV volume and mass, as well as to assess myocardial fibrous involvement. However, this is an expensive methodology that requires specialized equipment as well as highly qualified personnel to analyze the data.

The scientific community is faced with the question of which biomarkers to use to identify the early stages of development of cardiac fibrosis, as well as how to assess the degree of progression of this pathological process. A key point in the recovery response after heart damage is activation of fibroblasts, which is aimed at preserving the structure and function of the heart. Progress in deciphering these processes needs to be directed in several directions. A study of fibroblasts in physiological and pathological conditions is necessary. We should also establish to what extent fibroblast activation leads to cardiac dysfunction, arrhythmogenesis and other adverse effects. From the wide range of studied molecules, determination of collagen synthesis markers such as PIIINP and PICP is now routine. Emphasis is also placed on a number of signaling molecules involved in the initiation and progression of cardiac fibrosis such as CTGF, TGF-beta 1, fibronectin, endothelin 1 (ET-1), IL-1 and others.

Development of fibrous response is associated with various predisposing factors; therefore, it is necessary to develop a strategy to identify those patients who react with ECM overproduction.

Thus, it will be possible to focus on the impact of different signaling pathways in order to modulate the profibrotic state. 


\section{Compliance with ethical standards}

\section{Acknowledgments}

The authors gratefully acknowledge the University Hospital "Virgin Mary" for funding this work

\section{Disclosure of conflict of interest}

The authors declare that there is no conflict of interests regarding the publication of this paper

\section{References}

[1] Nag A. Study of non-muscle cells of the adult mammalian heart: a fine structural analysis and distribution. Cytobios. 1980; 28: 41-61.

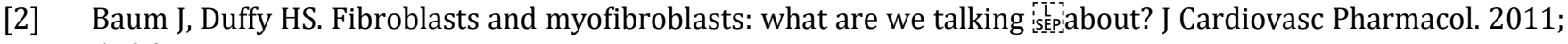
57(4): 376-379.

[3] Calderone A, Bel-Hadj S, Drapeau J, El-Helou V, Gosselin H, Clement R, Villeneuve L. Scar myofibroblasts of the infarcted rat heart express natriuretic peptides. J Cell Physiol. 2006; 207(1): 165-173.

[4] Fan D, Takawale A, Lee J' Kassiri Z. Cardiac fibroblasts, fibrosis and extracellular matrix remodeling in heart disease. Fibrogenesis \& Tissue Repair. 2012; 5: 15.

[5] Ambale-Venkatesh B, Lima JA. Cardiac MRI: a central prognostic tool in myocardial fibrosis. Nat. Rev. Cardiol. 2015; 12: 18-29.

[6] Hashimura H, Kimura F, Ishibashi-Ueda H, Morita Y, Higashi M, Nakano S, et al. Radiologic-pathologic correlation of primary and secondary cardiomyopathies: MR imaging and histopathologic findings in hearts from autopsy and transplantation. Radiographics. 2017; 37(3): 719-736.

[7] D'Armiento J. Matrix metalloproteinase disruption of the extracellular matrix and cardiac dysfunction. Trends Cardiovasc Med. 2002; 12(3): 97-101.

[8] Visse R, Nagase H. Matrix metalloproteinases and tissue inhibitors of metalloproteinases: structure, function, and bio- chemistry. Circ Res. 2003; 92(8): 827-839.

[9] Zannad F, Rossignol P, Iraqi W. Extracellular matrix fibrotic markers in heart failure. Heart Fail Rev. 2010; 15: 319-329.

[10] Holbourn K, Acharya K, Perbal B. The CCN family of proteins: structure-function relationships. Trends Biochem Sci. 2008; 33(10): 461-473.

[11] Shi-wen X, Leask A, Abraham D. Regulation and function of connective išẹtissue growth factor/CCN2 in tissue repair, scarring and fibrosis. Cytokine sisepiGrowth Factor Rev. 2008; 19: 133-144.

[12] Kubota S, Takigawa M. Cellular and molecular actions of CCN2/CTGF and its role under physiological and pathological conditions. Clin Sci (Lond) 2015; 128(3): 181-196.

[13] Cho Y, Silverstein R, Geisinger M, Martinkovich S, Corkill H, Cunnick J, et al. AFAP1 is a novel downstream mediator of TGF- $\beta 1$ for CCN2 induction in osteoblasts. PLoS One. 10(9): e0136712.

[14] Chen M, Lam A, Abraham J, Schreiner G, Joly A. CTGF expression is induced by TGF- beta in cardiac fibroblasts and cardiac myocytes: a potential role in heart fibrosis, J Mol Cell Cardiol. 2000; 32(10): 1805-1819.

[15] Chuva de Sousa Lopes S, Feijen A, Korving J, Korchynskyi O, Larsson J, Karlsson S et al. Connective tissue growth factor expression and Smad signaling during mouse heart development and myocardial infarction, Dev Dyn. 2004; 231(3): 542-550.

[16] Eschalier R, Rossignol P, Kearney-Schwartz A, Adamopoulos C, Karatzidou K, Fay R, et al. Features of cardiac remodeling, associated with blood pressure and fibrosis biomarkers, are frequent in subjects with abdominal obesity. Hypertension. 2014; 63: 740-6.

[17] Abed HS, Samuel CS, Lau DH, Kelly DJ, Royce SG, Alasady M, et al. Obesity results in progressive atrial structural and electrical remodeling: implications for atrial fibrillation. Heart Rhythm 2013; 10(1): 90-100. 
[18] Leichman JG, Aguilar D, King TM, Vlada A, Reyes M, Taegtmeyer H. Association of plasma free fatty acids and left ventricular diastolic function in patients with clinically severe obesity. Am J Clin Nutr. 2006; 84(2): 336-41.

[19] Cuspidi C, Rescaldani M, Sala C, Grassi G. Left-ventricular hypertrophy and obesity: a systematic review and metaanalysis of echocardiographic studies. J Hypertens. 2014; 32(1): 16-25.

[20] Zamani N, Brown CW. Emerging roles for the transforming growth factor-\{beta\} superfamily in regulating adiposity and energy expenditure. Endocr Rev. 2011; 32(3): 387-403.

[21] Verheule S, Sato T, Everett T, Engle SK, Otten D, Rubart- von der Lohe M, et al. Increased vulnerability to atrial fibrillation in transgenic mice with selective atrial fibrosis caused by overexpression of TGF-beta1. Circ Res. 2004; 94(11): 1458-65.

[22] Diez J. Mechanisms of cardiac fibrosis in hypertension. J Clin Hypertens (Greenwich). 2007; 9: 546-550.

[23] Nakahara T, Takata Y, Hirayama Y, Asano K, Adachi H, Shiokawa G, et al. Left ventricular hypertrophy and geometry in untreated essential hypertension is associated with blood levels of aldosterone and procollagen type III amino-terminal peptide. Circ J. 2007; 71: 716-721.

[24] Poulsen SH, Andersen NH, Heickendorff L, Mogensen C, et al. Relation between plasma amino-terminal propeptide of procollagen type III and left ventricular longitudinal strain in essential hyperten- sion. Heart. 2005; 91(5): 624-629.

[25] Alla F, Kearney-Schwartz A, Radauceanu A, Das Dores S, Dousset B, Zannad F. Early changes in serum markers of cardiac extra-cellular matrix turn- over in patients with uncomplicated hypertension and type II diabetes. Eur J Heart Fail. 2006; 8(2): 147-153.

[26] Löfsjögård J, Thomas K, Javier D, Begoña L, Arantxa G, Magnus E, et al. Biomarkers of collagen type I metabolism are related to B-type natriuretic peptide, left ventricular size, and diastolic function in heart failure. Journal of Cardiovascular Medicine. 2014; 15(6): 463-469.

[27] Lo' pez B, Gonza' lez A, D'iez J. Circulating biomarkers of collagen metabolism in cardiac diseases. Circulation. 2014; 121: 1645-1654.

[28] Loring Z, Zareba W, McNitt S, Strauss DG, Wagner GS, Daubert JP. ECG quantification of myocardial scar and risk stratification in MADIT-II. Ann. Noninvasive Electrocardiol. 2013; 18: 427-435.

[29] Lopez B, Gonzalez A, Ravassa S, Beaumont J, Moreno MU, San Jose G, et al. Circulating biomarkers of myocardial fibrosis: the need for a reappraisal. J. Am. Coll. Cardiol. 2015; 65: 2449-2456.

[30] Chiao YA, Ramirez TA, Zamilpa R, Okoronkwo SM, Dai Q, Zhang J, Jin YF, Lindsey ML . Matrix metalloproteinase9 deletion attenuates myocardial fibrosis and diastolic dysfunction in ageing mice. Cardiovasc Res. 2012; 96(3): 444-455.

[31] Kannel WB, McGee DL. Diabetes and cardiovascular disease. The Framingham study. JAMA. 1979; 241(19): 20358.

[32] Xu Z, Sun J, Tong Q, Lin Q, Qian L, Park Y, et al. The Role of ERK1/2 in the Development of Diabetic Cardiomyopathy. Int J Mol Sci. 2016; 17(12).

[33] Li CJ, Lv L, Li H, Yu DM. Cardiac fibrosis and dysfunction in experimental diabetic cardiomyopathy are ameliorated by alpha-lipoic acid. Cardiovasc Diabetol. 2012; 11: 73.

[34] Lovic D, Erdine S, Catakoglu AB. How to estimate left ventricular hypertrophy in hypertensive patients. The Anatolian journal of cardiology. 2014; 14: 389-95.

[35] Chaudhary AK, Aneja GK, Shukla S, Razi SM. Study on Diastolic Dysfunction in Newly Diagnosed Type 2 Diabetes Mellitus and its Correlation with Glycosylated Haemoglobin (HbA1C). Journal of Clinical \& Diagnostic Research. $2015 ; 9(8)$.

[36] Suematsu Y, Miura SI, Goto M, Matsuo Y, Arimura T, Kuwano T, et al. LCZ696, an angiotensin receptor-neprilysin inhibitor, improves cardiac function with the attenuation of fibrosis in heart failure with reduced ejection fraction in streptozotocin-induced diabetic mice. European Journal of Heart Failure. \& Pharmacology. 2016; 109: 321-31.

[37] Zhang F, Dang Y, Li Y, Hao Q, Li R, Qi X. Cardiac Contractility Modulation Attenuate Myocardial Fibrosis by Inhibiting TGF- $\beta 1 /$ Smad3 Signaling Pathway in a Rabbit Model of Chronic Heart Failure. Cellular Physiology \& Biochemistry International Journal of Experimental Cellular Physiology Biochemistry. 2016; 39: 294-302. 